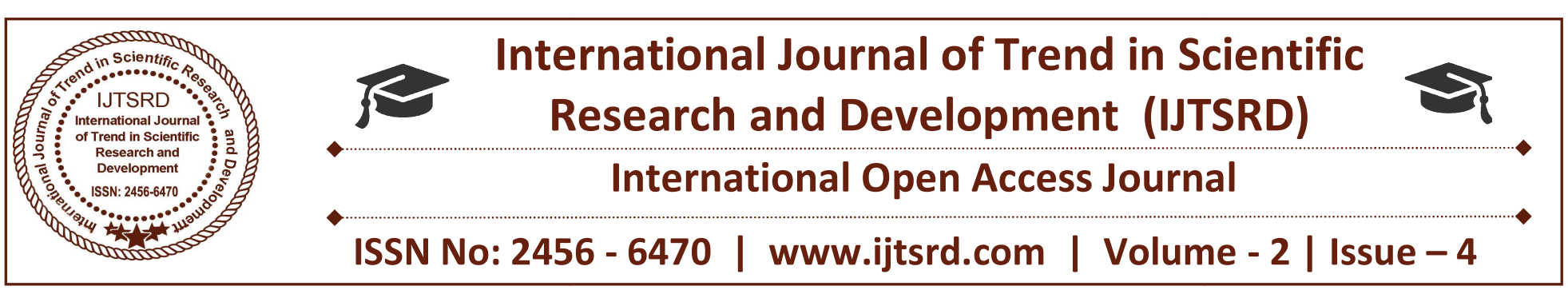

\title{
Structural Health Monitoring (SHM) of Highway bridges using Wireless Sensor Network
}

\author{
Pooja Bhadane, Akanksha Mali, Mukta Fulse, Jayashri Patil, Prof. S. B. Wagh \\ Department of Computer Engineering, Late G.N.SAPKAL College of Engineering, \\ Nasik, Maharashtra, India
}

\begin{abstract}
The objective is to provide a solution to measure vibrations, strain inside a concrete structure. Using Wireless sensor network it remotely monitor health of bridges. The goal of the monitoring is understand the structure \& prevent the damage \& prepares to repair it before catastrophe failure. The accelerometer, gas sensor, load cell and ultrasonic sensor module in existing sensor nodes enables several novel applications. In this paper, a model for monitoring and detecting damage for real bridge using these sensor nodes is built. The sensors are placed on a scaled down concrete bridge model i.e. mounted on a shaking table. The results are denoted in terms of acceleration on different nodes at a particular excitation frequency in the case of normal, single-side and double-side damage. The recommended system consists of a wireless Data Acquisition Unit (DAQ). The sensors in the DAQ assemble the bridge health signs and transmit them directly via the public mobile networks to the management and evaluation middleware for further processing. SHM system incorporates four major components: a wireless sensor system, a data processing system, alert system and a health evaluation system. This paper recommends a system architecture for smart healthcare based on GSM.
\end{abstract}

Keywords: Structural health monitoring (SHM), wireless sensor network(WSN), data acquisition unit(DAQ), global system for mobile(GSM)

\section{INTRODUCTION}

Monitoring structure condition have become a big challenge for engineers and researchers. The main principle of SHM is vibration analysis. Structural

Health Monitoring (SHM) is a essential tool to improve the safety and maintainability of critical structures such as bridges. SHM is able to monitor the structure of real time at evaluate the performance under many nodes \& determine damage of structure. Data acquired from the sensor is transferred through wireless network to destination. The goal of monitoring is understand the structure $\&$ prevent the damage \& prepares to repair it before harmful failure .Though a certain amount of data loss is sufficient in many SHM applications, more reliable data transmission is always to provide more accurate analysis based on the data.SHM process incorporates many sensors such as, accelerometer, gas, load cell, ultrasonic and gyroscope. Cloud platform allows the SHM data to be stored and used intelligently for smart monitoring and actuation with smart devices. GSM services are used for global communications anytime and anywhere. SHM system have excellent potential to improve the regular operation $\&$ maintenance of structure.

GSM is used for secured wireless communication. GSM modules can be placed in both sensing unit \& server unit. For wireless module this system is using GSM module \& output module is worked on pc. Receiver section is advanced using GSM module \& PC. The GSM module transmits vibration information, SIM number, date, time etc. to the main machine. The Central server's GSM module receives vibration information via SMS. To measure this vibration accelerometer is used. Accelerometer is type of sensor that can measure the structural vibration. The stability and usability of Wireless Sensor Network (WSN) is validated due to merits as such 
low cost, easy installation, and effective data management.

DAQ is the process of measuring an electrical or physical aspect similarly voltage, current, temperature, pressure, or sound with a computer. A DAQ system subsist of DAQ measurement hardware, computers with programmable software, and sensors.

\section{OBJECTIVE}

It checks ill condition of bridges, Sending the status of all parameters using GSM Module to Mobile Receiver of the authenticated person located anywhere. If status of any parameters is beyond the specified limit then it will generate the alarm. System sends the information using mobile network to operator to take appropriate actions before any damage occurs.

\section{LITERATURE SURVEY}

Some failures are sudden, harmful and some failures just take their time. Structural Health Monitoring (SHM) can be very helpful in serving as an generating alert for preventing various types of failures. Bridge Engineers demands scientific tools which can give quick information about the health of a bridge. Such instrument shall added feature the periodical manual inspections. But when failures happen with any kind of structure there is loss of human lives, money and many more, most of the times. For example, during the bridge construction burst of the 1950's and 1960's, little emphasis was placed on safety inspection and conservation of bridges. This transposed when the 2,235 foot Silver Bridge at Point Cordial, WV, crumpled into the Ohio River, on Dec. $15,1967.46$ public were assassinated.

Hence to ensure the safety of bridges, the Bridge Health Monitoring System was popularized. Some of the existing technologies/methods for Bridge Health Monitoring System are as described.

1.Mr. M. V. N. R. P. Kumar, Miss. J. D. Kadam, Ms. B. Hombal, Mr. B. M. Pawar, Mr. A. B. Yadav." Bridge Condition Monitoring System Using PIC Microcontroller":A multi-functional wireless bridge monitoring system has been developed for concurrent deployment of accelerometers, strain transducers, temperature sensors and anemometer. The sensing capabilities of these nodes satisfies the immediate requirements for economic, low-maintenance load ratings and short-term dynamic measurements in addition to providing the hardware functionality for development of a long-term continuous bridge monitoring system. Comprehensive laboratory and field testing and development has been performed to produce a reliable radio transmission protocol capable of preserving a large number of nodes with high data throughput in real-time[1].

2. Chae, M. J. Ph. D, P. E. Kim, Yoo H.S.J.R. Cho, M. Y, Ph. D."Bridge condition monitoring system using wireless network (CDMA and zigbee)": As per with the help of the wireless technology many problems due to data cables and

expensive optical cable are now minimized and excluded. Sensor and ZigBee module mingled becomes u-node (ubiquitousnode). ZigBee is proved to be excellent solution in short distance wireless data communication. For long distance. data transferring CDMA which is a mobile phone carrier network in Korea is used rather than optical cable which is expensive in installation and maintenance[2].

3.Michael P. Fuchs,Michael V.Gangone,Matthew J. Whelan, Clarkson University, "Development of a wireless bridge monitoring system for condition assessment Using hybrid techniques": A multifunctional wireless bridge monitoring system has been developed for concurrent formation of strain transducers, temperature sensors and accelerometers. The hybrid sensing potentials of these node comforts the prompt requirements for commercial, flat conservation load ratings and short-term dynamic measurements in addition to providing the hardware functionality for development of a long-term repeated monitoring system of bridge[3].

\section{SYSTEM ARCHITECTURE}

The system architecture interprets the comprehensive configuration of the complete system. It scrutinizes the monitoring of bridge for mainly following parameters in order to avoid risky effects: 1 . Vibrations, 2. Fire in vehicle, 3. Water level 4.Load on the bridge. So as to have a repeated health monitoring of bridge and identify any damage being made to it so as to take precautionary measures in prior this system has installed various sensors on divergent locations. Data (measures parameters load, vibrations, and Motion) seized from these sensor is conditioned and then sent to controller which further sends it to GSM module which later transmit it to the GSM receiver at remote location. A alert generates as soon as the limit exceeded. Actuator is being used to take immediate disciplinary action in situation of 
emergencies. Establish WSN across the bridge to pickup vibration and sound data from various parts. Send WSN data to the cloud. Generate notification for irrigation, state transport, hospital, police, fire brigade regarding damage of bridge. Display a list of ill condition bridges (status of bridges). The sensors used for monitoring the health of bridges and their working is as follows:

\section{Gyroscope:}

The MPU-60X0 is the world's first unified 6-axis Motion Tracking device that combines a 3-axis gyroscope, and a Digital Motion Processor ${ }^{\mathrm{TM}}$ (DMP) all in a small $4 \times 4 \times 0.9 \mathrm{~mm}$ package. With its devoted I2C sensor bus, it precisely gets inputs from an external 3-axis compass to provide a complete 9-axis output of Motion Fusion ${ }^{\mathrm{TM}}$. The MPU60X0 Motion Tracking device, with 6-axis assimilation, on-board Motion Fusion ${ }^{\mathrm{TM}}$, and run-time positioning firmware, empowers manufacturers to exclude the pricey and convoluted selection, qualification, and system level assimilation of discrete devices, for consumers assures optimal motion achievement. The MPU$60 \mathrm{X} 0$ is also designed to interface with multiple non inertial digital
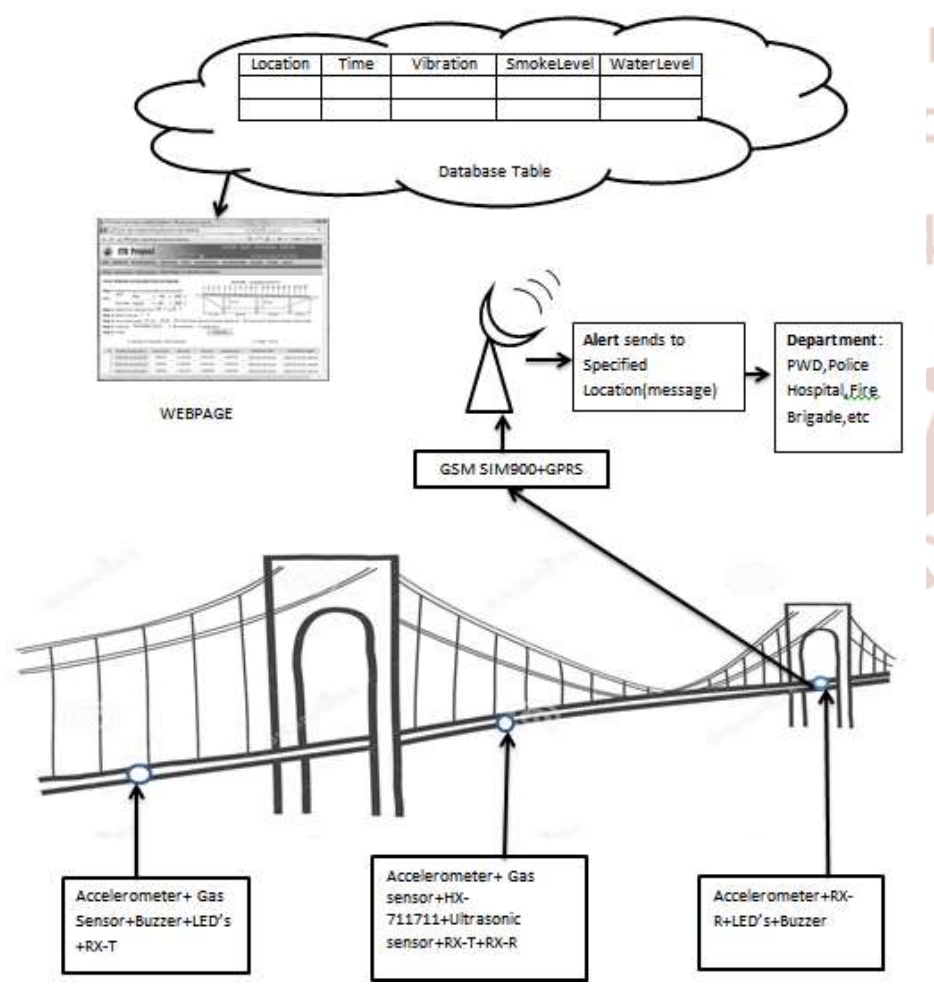

Fig: System Architecture sensors, like pressure sensors, on its ancillary I2C port. The MPU-60X0 is footprint consistent with the MPU-30X0 family. The MPU-60X0 features three 16-bit ADC converters(s) for computerizing the gyroscope outputs.

\section{Accelerometer:}

Accelerometers have been extensively used as a measuring device for dynamic aspect for many years. These devices determine acceleration at a given instant and they can sample at high frequencies to yeild high-resolution time histories of acceleration. The acceleration matters a known mass to spawn a force in the sensor creating a small current or a change in the current. Since the force and mass are known, then the acceleration can be accessed by proper adjustment. Using the acceleration versus time data, a displacement (or velocity) history can be accessed by numerical integration. These devices spawn large amounts of data, so intensive processing is necessary to get an accurate picture of deflection. One aspect on the use of accelerometers is that they can provide useful measurements.

\section{Strain Sensor:}

strain gauges are sensing devices that change resistance at their output terminals when reduced. They are typically secured to the surface of a solid material to measure its minute dimensional changes when put into compression or tension. Strain gauges and their concealed principles are usually used in devices for measuring pressure, acceleration, force, and tension.

\section{RF:}

An RF module (radio frequency module) is a (usually) small electronic appliance used to broadcast and/or receive radio beacon among two devices. In an embedded system it is usually enticing to interact with another device wirelessly. This wireless communication may be polished through optical transmission or over radio frequency (RF) transmission. RF modules are most ordinarily used in low and intermediate variety brands for user applications such as garage door passkey, wireless alarm or supervising systems, industrial isolated controls, smart sensor applications, and wireless home computerization systems. They are frequently used to change earlier infrared transmission layout as they have the advantage of not demanding line-of-sight operation. Different carrier frequencies are commonly 
used in commercially feasible RF modules, counting those in the industrial, medical and scientific (ISM) radio bands such as $433.92 \mathrm{MHz}, 915 \mathrm{MHz}$, and $2400 \mathrm{MHz}$. These frequencies are used in behalf of national and international regulations govern the used of radio for communication. Brief scope Devices may also use frequencies reachable for illicit such as $315 \mathrm{MHz}$ and $868 \mathrm{MHz}$.

\section{Ultra Sonic Sensor:}

Ultrasonic sensor is one of the most commonly used sensor for span analysis. It works on the principal of ultrasound i.e. it radiate the waves whose frequency is higher than $20 \mathrm{KHz}$ which is not detectable to human ear and it is typically ranging up to $40 \mathrm{KHz}$. In Ultrasonic sensor, it subsist of two lenses, transmitter and receiver. The transmitter (Trigger pin) broadcast the ultrasound wave, the wave grasp till the object and divert back. The receiver (Echo pin) accept the diverted wave and the period of this travelling of waves is deliberated by the Arduino and transformed into definite bulk of time in Microseconds.

\section{Gas Sensor:}

In the air the various gases and there mixture are exists(oxygen, carbon dioxide, hydrogen ).In that, some hazardous gases are also exist. A gas detector is a device that detects the presence of gases in an area, often as part of a safety system. The MQ-2 is a flammable gas and smoke sensor detects the concentrations of combustible gas in the air. The sensor can measure concentrations of flammable gas of 300 to $10,000 \mathrm{ppm}$. In the gas sensor there is a heater is used(circuit for converting the gas concentration into voltage value).The MQ-2 gas sensor is sensitive to LPG, i-butane, propane, methane, alcohol, Hydrogen and smoke. When target combustible gas exist, in the sensor's due to conductivity is increases as the gas concentration rising. As the conductivity is increases the temperature of heater will increase and hence resistance. This resistance can convert into voltage and this signal is given to Arduino. This type of material is used to catch a gas leak and bind with a control system so a operation can be automatically shut down.

\section{PROPOSED SYSTEM}

The main aim of this proposed system to overcome the problem of manual health monitoring of bridges. In this proposed system the monitoring health of bridge is automated at remote places using wireless sensor networks, micro controller and GSM. Vibration, Gas Level, smoke level, weight etc is the parameters that are analyzed to improve the health of bridge. Following are the objectives of idea implementation :

To provide a solution to measure vibration, load and strain inside a concrete structure.

$>$ To check ill condition of bridge

$>$ To reduce accident

$>$ To collect data from the given sensors and send it to base station and the server(data base) or Irrigation department or PWD, State transport department, hospital by GSM module.

To send notification to an authorized person automatically when bridge health is degraded and it does not match the given standards.

The detailed block diagram of Structural health monitoring of highway Bridges using WSN is shown in Fig:

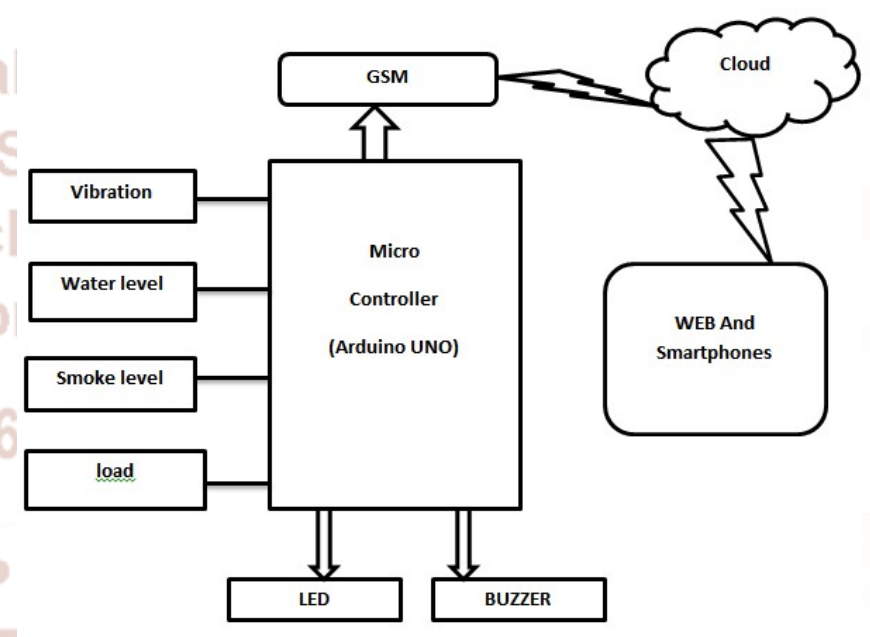

Fig. Block Diagram

\section{ADVANTAGES}

It checks ill condition of bridges

$>$ It reduce \& prevent accidents

$>$ Monitor condition of bridges

$>$ Uses structure flexibility matrix to localize structural damage

$>$ Data analysis

> Improves scalability by using multi-level time synchronization Approach

$>$ Easy to implement

$>$ Increasing reliability, safety \& efficiency 


\section{EXPERIMENTAL RESULT}

Several Bridges from different places were examined to establish a reference on the parameters for each bridge type.Thesebridges health were tested simultaneously. Readings were taken at 1 hour intervals for a total period of 12 hours. Here we are showing the results of only one bridge with respect to time.

1.The following figure shows the vibration generated on bridge due to earthquake or accident w.r.to time.

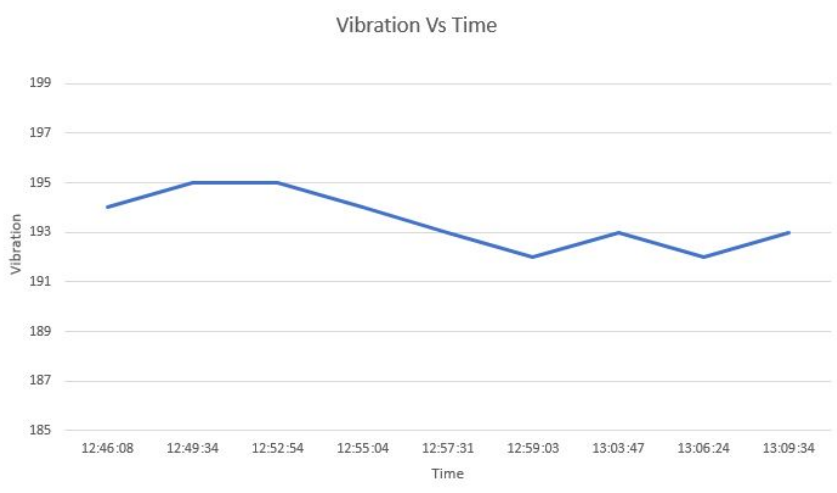

2.The following figure shows the Tilt angle of bridge due to earthquake or other disaster w.r.to time.

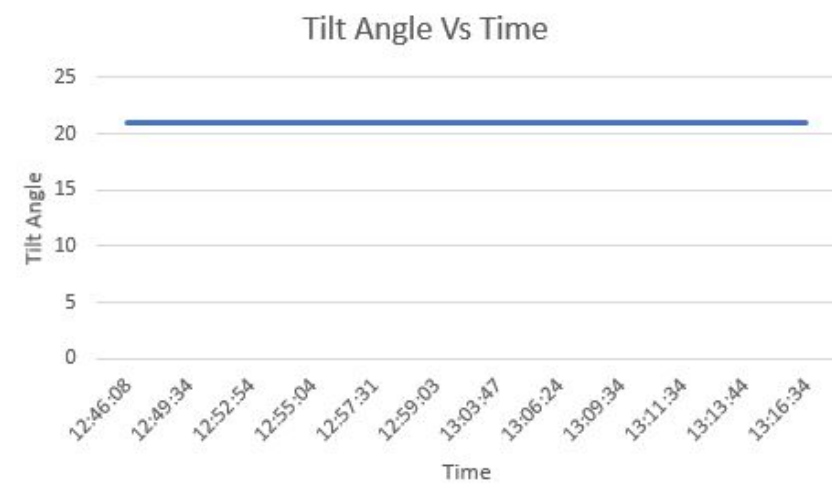

3. The following figure shows the smoke generated on bridge due to fire in vehicle or fire on bridge w.r.to time.

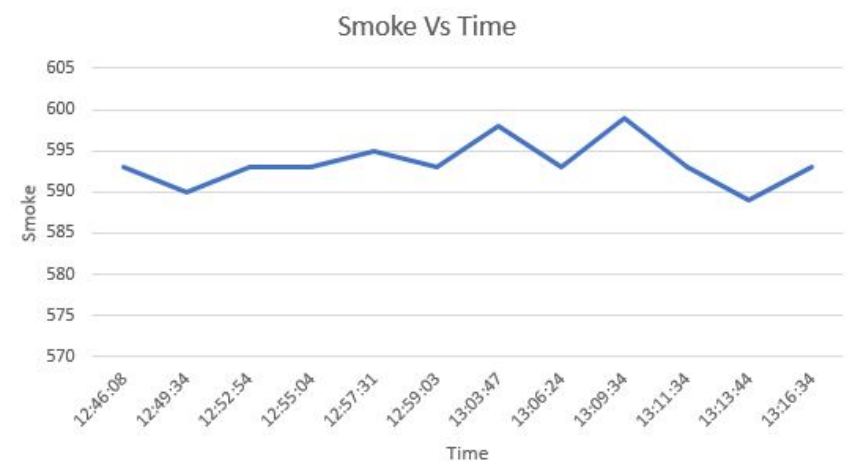

4. The following figure shows the water levelunder the bridge w.r.to time.

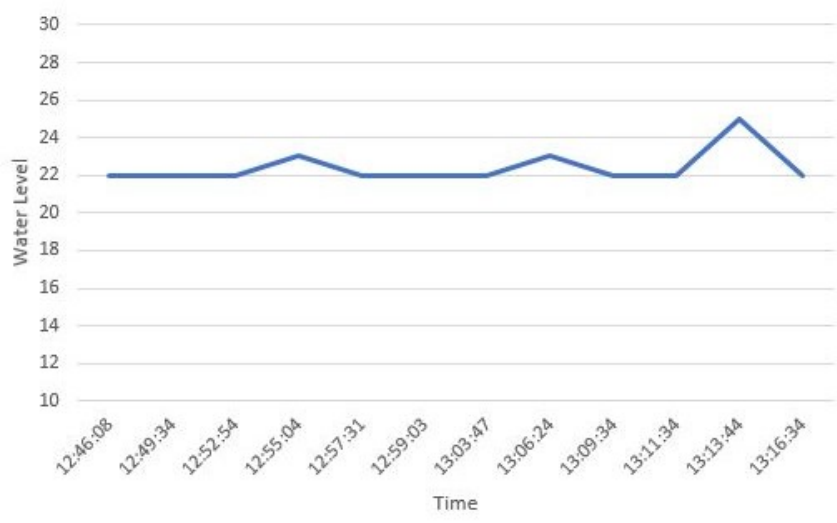

5.The following figure shows the weight of vehicle that is passing from the bridge w.r.to time.

\section{Weight Vs Time}

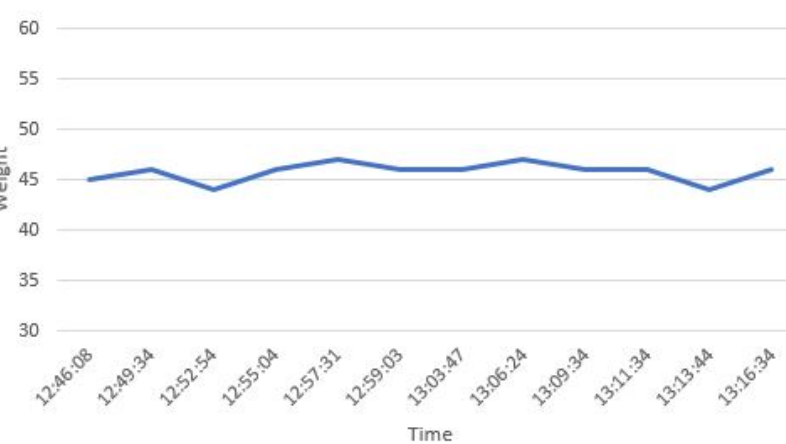

IMPLEMENTATION AND OUTPUT

As per given system architecture and modules, the system is proving a better results for avoiding the accidents on bridge. This project defines the use of IoT in the field of PWD department for performing various task in this field without Human interaction. In this system, we created WSN network. In this system there are 3 nodes first is sensor node, second is router node, third is co-ordinator node, and at the last there is GSM. From these nodes the readings are taken and send to the website for displaying to the authorised user. These output is displayed in table format as given below. In this table, there is nodeID which is the id given to the nodes in the network. Vibration field gives the vibration reading in case of earthquake. Tilt Field detects the accident and smoke value detects if there is fire in vehicle or on the bridge. Water Level monitors the water level if the bridge is on the river. Weight field gives the weight of vehicle that is passing from the bridge. 


\begin{tabular}{l|l|l|l|l|l|l|l|l} 
ID & $\begin{array}{l}\text { Node } \\
\text { Id }\end{array}$ & $\begin{array}{l}\text { Vibrati } \\
\text { on }\end{array}$ & Tilt & $\begin{array}{l}\text { smoke } \\
\text { value }\end{array}$ & $\begin{array}{l}\text { water } \\
\text { Level }\end{array}$ & weight & Date & Time \\
\hline 1 & 1 & 0 & 21 & 590 & 22 & 45 & $03-04-2018$ & $12: 46: 08$ \\
\hline 2 & 2 & 198 & 768 & 593 & 22 & 48 & $03-04-2018$ & $12: 49: 34$ \\
\hline 3 & 3 & 67 & 21 & 588 & 22 & 43 & $03-04-2018$ & $12-53: 42$ \\
\hline 4 & 1 & 12 & 21 & 588 & 22 & 45 & $03-04-2018$ & $12-58: 48$ \\
\hline 5 & 2 & 60 & 768 & 590 & 22 & 47 & $03-04-2018$ & $13: 03: 42$ \\
\hline 6 & 3 & 124 & 21 & 596 & 22 & 49 & $03-04-2018$ & $13: 06: 51$ \\
\hline
\end{tabular}

\section{CONCLUSION}

In this system, the model of structural health of bridge is monitored using Internet of Things technology is presented.

Bridge health condition monitoring has been a prominent\& essential problem. This project serve as a new SHM system that is being used to monitor and alert system of bridges. Here a wireless sensor network (WSN) system is advanced. With the help of the wireless technology many problems due to data cables and expensive optical cable are now underestimate. The GSM transmission comforts in better way of communication which provides data transmission adequately between the GSM module and receiver. The generated data can be viewed using web interface all over the world to the authenticated department.

\section{REFERENCES}

1) Mr.M.V.N.R.P.Kumar,Ms.B.Hombal, Miss. J.D. Kadam, Mr. A. B. Yadav, Mr. B.M. Pawar." Bridge Condition Monitoring System Using PIC Microcontroller"

2) Chae, M.J.Ph.D, P.E.Kim, Yoo H.S.J.R. Cho,M.Y,Ph. D.'Bridge condition monitoring system using wireless network (cdma and zigbee)"

3) Matthew J. Whelan, Michael P. Fuchs, Michael V.Gangone, Clarkson University, "Development of a wireless bridge monitoring system for condition assessment Using hybrid techniques"

4) Sodano, H. A., Inman, D. J. And Park, G., A Review Of Power Harvesting From Vibration Using Piezoelectric Materials. Shock Vib. Digest, 2004, 36, 197-205.

5) Paek, J. Et Al., The Performance Of A Wireless Sensor Network For Structural Health Monitoring. University Of Southern California And University Of California Los Angeles, Los Angeles, California, USA, 2005. 1

6) Ilyas, M. And Mahgoub, I. (Eds),Handbook Of Sensor Networks: Compact Wireless And Wired Sensing Systems, CRC Press, 2005.
7) Kim, S., Pakzad, S., Culler, D., Demmel, J., Fenves, G., Glaser, S. And Turon, M., Health Monitoring Of Civil Infrastructures Using

8) Wireless Sensor Networks. In IPSN'07, Cambridge, Massachusetts, USA, 25-27 April 2007.

9) Kiremidjian, A.S., Kenny, T.W., Law, K.H. \& Lee, T. 2001. "A Wireless Modular Health Monitoring System For Civil Structures." Proposal To The National Science Foundation, NSF 0121842.

10) Lynch, J.P., Law K.H., Kiremidjian A.S., Kenny T.W., Carryer E. \& Partridge A. 2001. "The Design Of A Wireless Sensing Unit For Structural Health Monitoring." 3rd International Workshop On Structural Health Monitoring, Stanford, CA, Pp. 1041-1050.

11) Dhivya A\#1, Hemalatha. M*2 \#M.TechEmbedded Systems, SASTRA University Thanjavur, Tamil Nadu, India Assistant Professor, SASTRA University Thanjavur, Tamil Nadu, India "STRUCTURAL HEALTH MONITORING SYSTEM - AN EMBEDDED SENSOR APPROACH" International Journal Of Engineering And Technology (IJET) ISSN : 09754024 Vol 5 No 1 Feb-Mar 2013. 